INPLASY

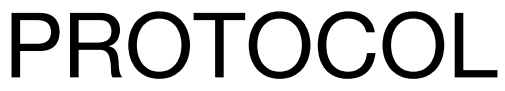

To cite: Qiu et al. Efficacy and Safety of Acupotomy Release in Treating Upper Cross

Syndrome: A protocol for systematic review and metaanalysis. Inplasy protocol

202170012. doi:

10.37766/inplasy2021.7.0012

Received: 05 July 2021

Published: 05 July 2021

Corresponding author:

Mingquan Wei

282344043@qq.com

Author Affiliation:

The Affiliated Hospital of Jiangxi University of Traditional Chinese Medicine Jiangxi University of Traditional Chinese Medicine

Support: 2019XZZX-LG005.

Review Stage at time of this submission: The review has not yet started.

Conflicts of interest:

None declared.

\section{Efficacy and Safety of Acupotomy Release in Treating Upper Cross Syndrome: A protocol for systematic review and meta-analysis}

\author{
Qiu, WC1; Wei, MQ2; Zhang, P3; Li, RL44.
}

Review question / Objective: Upper cross syndrome (UCS) is a condition in which postural abnormalities lead to mechanical imbalances in muscle groups, secondary to a "painful spasm cycle". Previous reviews of acupotomy release for UCS have been growing, but a systematic review is not available. This study aimed to systematically investigate the efficacy and safety of acupotomy release in treating UCS.

Eligibility criteria 1.Types of studies. RCTs on the effect of acupotomy release in treating UCS will be included in this study. 2.Types of participants. Patients who met the standard for UCS diagnosis will be included. We do not apply any restrictions in terms of age, gender, and ethnicity. 3.Types of interventions. The control group was treated with conventional therapy or other adjuvant therapy without acupotomy release therapy. The treatment group was treated alone with acupotomy release therapy, or acupotomy release combined with conventional therapy and other adjuvant therapy. 4.Outcomes. The total effective rate and visual analog scale (VAS) score are the primary outcome indicators of this study. This study's secondary outcome is as follows: neck disability index(NDI), Forward head angle(FHA), and Forward shoulder angle(FSA).

INPLASY registration number: This protocol was registered with the International Platform of Registered Systematic Review and Meta-Analysis Protocols (INPLASY) on 05 July 2021 and was last updated on 05 July 2021 (registration number INPLASY202170012).

\section{INTRODUCTION}

Review question / Objective: Upper cross syndrome (UCS) is a condition in which postural abnormalities lead to mechanical imbalances in muscle groups, secondary to a "painful spasm cycle". Previous reviews of acupotomy release for UCS have been growing, but a systematic review is not available. This study aimed to systematically investigate the efficacy and 
safety of acupotomy release in treating UCS.

Condition being studied: Upper cross syndrome (UCS) was first proposed by Vladimir Janda MD, a Czech medical rehabilitation expert, in 1988. UCS often presents with rounded shoulders, hunched back, and forward head tilts, mostly accompanied by neck and shoulder discomfort such as soreness and pain. Their postural abnormalities are caused by abnormal contraction of the pectoralis minor, superior trapezius, pectoralis major, scapular raphe, and sternocleidomastoid muscles, and weakness in stretching the muscles of the middle and lower trapezius, rhomboids, and serratus anterior. The abnormally contracted and stretched muscles form an " $X$ " in the upper part of the body. UCS is prevalent in the general population, including $28 \%$ of laundry workers between the ages of 25 and 50. In a survey of 101 Iranian office workers, $61.3 \%$, $48.7 \%$, and $78.3 \%$ exhibited forward head, backward chest, and rounded shoulder postures, respectively.The prevalence of neck and shoulder muscle imbalancerelated disorders fluctuates between $11 \%$ and $60 \%$ across societies and age groups, with an increasing trend over the years. UCS not only affects the patient's daily routine, but also has a negative impact on sleep and emotional functioning, while repeated visits to the doctor add to the financial burden of the patient. There is no uniform clinical standard for the treatment of UCS, so the investigation of effective treatment methods for upper crossed syndrome has become a clinical research hotspot.Acupotomy is a type of acupuncture used in traditional Chinese medicine (TCM), and it has both the characteristics of a "needle" in TCM and a "knife" in Western medicine. Compared to traditional acupuncture needles, acupotomy release therapy can not only dredge the meridians and regulate qi and blood by identifying and selecting meridian points, but also release the adhesions, scarring, contractures, blockages and other pathological changes in the surrounding soft tissues, restore the biodynamic mechanical balance between soft tissues, improve local microcirculation, eliminate muscle tension and muscle spasm, and achieve the therapeutic effect of relieving local pain and correcting abnormal posture. Evidence from RCTs has shown that acupotomy release is an effective way to treat UCS. There is still a lack of evidence-based medicine evidence to support it. In this study, we aimed to evaluate the efficacy and safety of acupotomy release for UCS objectively and provide reliable evidence for the clinical application of acupotomy release in UCS.

\section{METHODS}

Participant or population: Patients who met the standard for UCS diagnosis will be included.

Intervention: The treatment group was treated alone with acupotomy release therapy, or acupotomy release combined with conventional therapy and other adjuvant therapy.

Comparator: The control group was treated with conventional therapy or other adjuvant therapy without acupotomy release therapy.

Study designs to be included: Randomized controlled trials (RCTs) will be included in this review.

Eligibility criteria: 1.Types of studies. RCTs on the effect of acupotomy release in treating UCS will be included in this study. 2.Types of participants. Patients who met the standard for UCS diagnosis will be included. We do not apply any restrictions in terms of age, gender, and ethnicity. 3.Types of interventions. The control group was treated with conventional therapy or other adjuvant therapy without acupotomy release therapy. The treatment group was treated alone with acupotomy release therapy, or acupotomy release combined with conventional therapy and other adjuvant therapy. 4.Outcomes. The total effective rate and visual analog scale (VAS) score are the primary outcome indicators of this study. This study's secondary outcome is as follows: neck disability 
index(NDI), Forward head angle(FHA), and Forward shoulder angle(FSA).

Information sources: We will search candidate studies to evaluate the effect of acupotomy release in treating UCS through PubMed, Embase, the Cochrane Library, China National Knowledge Infrastructure (CNKI), Chinese Biomedical Literature Database(CBM), China Science and Technology Journal Database (VIP) and Wanfang Database until October 2021.

Main outcome(s): The total effective rate and visual analog scale (VAS) score are the primary outcome indicators of this study.

Additional outcome(s): This study's secondary outcome is as follows: neck disability index(NDI), forward head angle(FHA), and forward shoulder angle(FSA).

Quality assessment / Risk of bias analysis: Two reviewers independently assessed the risk of bias in accordance with the assessment tool suggested in the Cochrane Handbook for Systematic Reviews of Interventions. Seven domains including random sequence generation (selection bias), allocation concealment (selection bias), blinding of participants and personnel (performance bias), blinding of outcome assessment (detection bias), incomplete outcome data (attrition bias), selective reporting (reporting bias), and other bias were used for the methodological quality of each included trials. For all the relevant outcomes in the relevant domains, the quality of each item was classified using a nominal scale: low risk of bias, high risk of bias, or unclear risk of bias.

Strategy of data synthesis: The statistical analysis was performed by Review Manager 5.3 software (the Cochrane Collaboration, Copenhagen, and the Nordic Cochrane Centre). For measurement data, dichotomous variables were presented as risk ratio (RR), while continuous outcomes were presented as the mean difference (MD) or standard mean difference (SMD) with $95 \%$ confidence intervals (Cls). The I- square (12) statistic was used to assess heterogeneity. If $P>0.1,12<50 \%$, indicating small heterogeneity; the fixed effect model was used for meta-analysis. If $12>50 \%, P<0.1$, it indicated that there was a high degree of heterogeneity among the study results; then, the random effect model was applied. The source of heterogeneity was analyzed by subgroup analysis. Sensitivity analysis was used for the stability of the analysis results. Whether bias occurs or not was indicated by the funnel plot.

Subgroup analysis: If the heterogeneity source cannot be found after sensitivity analysis, we will perform further subgroup analysis.

Sensitivity analysis: We will use the leaveone-out method for sensitivity analysis to judge the stability of outcome indicators.

\section{Country(ies) involved: China.}

Keywords: Upper cross syndrome; acupotomy release; systematic review; protocol.

Contributions of each author:

Author 1 - Wencai QIU - The author drafted the manuscript.

Email: 1021395251@qq.com

Author 2 - Mingquan WEI - The author provided statistical expertise.

Email: 282344043@qq.com

Author 3 - Ping ZHANG - The author contributed to the development of the selection criteria, and the risk of bias assessment strategy.

Email: 1749743784@qq.com

Author 4 - Renliang Li - The author read, provided feedback and approved the final manuscript.

Email: 1051545802@qq.com 